\title{
The Influence of Respiratory Syncytial Virus on Breastfeeding and Maternal- Infant Bonding
}

\author{
Emily Tennenhouse ${ }^{1}$ \\ ${ }^{1}$ Arthur Labatt Family School of Nursing, Western University
}

\begin{abstract}
Breastfeeding is an integral element for facilitating long term immunity and bonding between a baby and their mother. When an infant is infected with Respiratory Syncytial Virus (RSV) they are put at risk of easy tiring, which increases the likelihood of requiring nutritional supplementation from a bottle to maintain adequate nutrition and hydration. This review article will discuss the pathophysiology behind RSV, how RSV can impair successful breastfeeding, the social determinants of health related to RSV and breastfeeding, and recommendations to upkeep exclusive breastfeeding if an infant has RSV to facilitate mother-infant bonding.
\end{abstract}

KEYWORDS: breastfeeding, respiratory syncytial virus, lactation, bonding, social determinants of health, nursing

\section{Introduction}

Respiratory syncytial virus (RSV) is a lower respiratory infection which accounts for more than $60 \%$ of acute respiratory infections in children of all ages and approximately $80 \%$ of lower respiratory tract infections in infants younger than 1 year of age (29). For years breastfeeding has been associated with positive health outcomes for children (6). Some of the benefits to breastfeeding infants include but are not limited to, long-term protection from chronic illnesses, positive cognitive development, lower incidence of type 1 and type 2 diabetes and decreased incidence of obesity (6). Breastfeeding has also been shown to improve the facilitation of mother-infant bonding (22). Unfortunately, some infants with respiratory illnesses such as RSV are not able to exclusively breastfeed without intervention from a healthcare professional proficient in breastfeeding assistance. A common barrier encountered by mothers attempting to breastfeed their infant with RSV is easy tiring of the infant from energy exertion involved in breathing with a respiratory infection (24). Since milk flow through bottle feeding travels at a higher rate than from the breast, some women may have more success with bottle feeding their infant with RSV rather than breastfeeding their infant (26). This is because more milk can be fed to the infant at once and the infant will need to feed for a shorter period of time to receive the same amount of milk as with breastfeeding. This shorter feed time can often prevent energy depletion. Women can become tempted to quit exclusive breastfeeding because of this "quick-fix". However, nonexclusive breastfeeding for the first 6 months of an infant's life depletes the various benefits of breastmilk consumption (33). This paper will discuss background information on RSV and breastfeeding, how RSV can impair breastfeeding and maternal-infant bonding, the social determinants of health related to RSV and breastfeeding, and recommendations to upkeep exclusive breastfeeding if an infant has RSV to facilitate mother-infant bonding.

\section{Background Information and Current Perspectives}

Lower respiratory tract infections are the leading cause of morbidity and mortality in children (30) and when a child is infected with RSV, their parents may experience high levels of 
stress and anxiety related to their child's wellbeing.

The RSV pathogen belongs to the Pneumovirus genus, is encased within an envelope, and has a single-stranded RNA genome (20). The mode of transmission of RSV is through contact with respiratory droplets (25). This means that when someone with RSV coughs or sneezes, the respiratory droplets produced by these actions can cause infection to those who breathe in the droplets. The RSV pathogen infects the lower respiratory tract and causes the airway to narrow due to excess mucous production, and results in obstruction of the bronchioles from irritation (19). Children who have been in contact with droplets from an infected case may begin to show symptoms of RSV within four to six days post contact (36). The symptoms of RSV in children over six months of age include runny nose, decreased appetite, coughing, sneezing, fever, and wheezing. Children under six months of age will primarily present with irritability, decreased activity, and breathing difficulties (36). While most cases of RSV pass within a couple weeks, RSV can sometimes progress into a potentially serious condition called bronchiolitis (36). This complication is especially common in children under six months of age (36). Bronchiolitis includes a variation of clinical signs and symptoms such as early symptoms of an upper respiratory viral infection followed by increased respiratory effort and wheezing (1). The symptoms of bronchiolitis can range from dry cough, apneic periods, wheezing, and irritability to rapid breathing, high fever, dehydration, pneumonia, low oxygen levels, abnormal feeding, and respiratory failure (40), (38). When a child has bronchiolitis, they may not be able to maintain adequate oxygenation due to apnea, mucus plugging, respiratory distress, or lower airway obstruction (1). Hospitalization is common in children with bronchiolitis due to the potential for dehydration and/or difficulty breathing (36). In a study of the proportion of infants hospitalized with RSV and RSV related illness in Ontario between 2010 and 2011 it was found that of the 1,411 admissions of infants with RSV related illness, including bronchiolitis, 327 children were hospitalized (30). Improvements in pediatric intensive care have decreased the incidence of mortality from bronchiolitis, but children may still experience severe symptoms of bronchiolitis for up to four weeks (43).

Breastfeeding in relation to maternal-infant bonding is a new area of research. When human babies are born they are very dependent on their parents, and experience rapid brain growth and development within the first two years of their life (42). Early brain development in children is positively impacted by love and bonding with a parent or guardian, and one major opportunity to exercise bonding is through breastfeeding (42). One of the main reasons that breastfeeding is a time of bonding is because mothers produce high levels of the hormone oxytocin when they breastfeed (37). Oxytocin can decrease levels of anxiety in the mother, and this can help to facilitate bonding (37). Skin-to-skin contact is another bonding enabler involved in breastfeeding. Skin-to-skin contact is when a mother holds her nude baby against her bare skin (37). This action can increase levels of maternal responsivity, increase levels of oxytocin produced in the mother, and increase maternal-infant bonding (37).

\section{The Problem of}

\section{Respiratory Infection and Breastfeeding}

Just like when an adult eats, an infant requires the coordination of breathing and swallowing in order to maintain homeostasis within the body. An infant with RSV or RSV related illnesses can experience difficulty breathing and tiring from overuse of respiratory accessory muscles such as the scalene, sternocleidomastoid and external intercostal muscle groups (24). Accessory muscle use in breathing is associated with respiratory distress as it can unconsciously help to improve ventilation when normal breathing is insufficient (13). Combined with a decrease in appetite (24), easy tiring stemming from RSV infection can impact breastfeeding. The infant may not be able to stay on the breast for a long enough time to retrieve an adequate amount of milk from the nipple as it may need to stop to catch its breath. 
This complication is supported by investigating the oxygen levels of the infant. Studies have found that when an infant has a blood oxygen saturation level of under $92 \%$ or a respiration rate greater than 60 70 breaths per minute, they can have trouble feeding because of respiratory distress (27), (1).

Many hospitals in Canada have cut funding for lactation consultants and other forms of breastfeeding assistance (3). Without breastfeeding assistance in times of need, the mothers of RSV infected children may be inclined to switch to formula feeding their babies to ensure they receive adequate nutrition. When a mother is unable to breastfeed her infant, feelings of stress and inadequacy can occur for the mother. There is such an emphasis in modern society that "breast is best," that when a mother begins to struggle with breastfeeding, they believe that they are the root of the problem. Healthcare professionals have even contributed to these feelings of stress in breastfeeding mothers. There have been reports that current breastfeeding advocacy by healthcare providers can be overbearing and that it contributes to feelings of guilt and shame (5). Unfortunately, stress experienced by the mother can inhibit the letdown reflex of milk. The let-down reflex also known as the milk ejection reflex is a neuro-endocrine reflex triggered by stimulation of the nipple and areola (39). The infant will suck on the mother's nipple, which will trigger the reflex let-down reflex pathway to produce and eject breastmilk from the milk ducts (39). The limitation of the let-down reflex from stress in this circumstance further decreases the effectiveness of breastfeeding an infant with RSV (34). Additionally, the literature shows that numerous new mothers have feelings that they are inadequately feeding their child by breastfeeding, or that breastfeeding in itself is too challenging to be maintained on a long-term basis (32). These thoughts and feelings are some of the main causes of early cessation of exclusive breastfeeding (12). As such, if a mother is already thinking about quitting exclusive breastfeeding when there is no external factors inhibiting it, the additional challenge of attempting to breastfeed a child with RSV may be what finalizes the decision to begin formula feeding.

\section{The Social Determinants of Health and RSV}

The social determinants of health play a material role in determining which children are at the highest risk for attaining RSV. The social determinants of health can also negatively impact exclusive breastfeeding and maternal-infant bonding.

Income is recognized as a social determinant of health by the Government of Canada (10). Studies have found that living in a home with no sewage or running water was significantly associated with attaining RSV (14). Persons with low-income are more likely to live in sub-optimal housing environments without running water or sewage compared to their high-income counterparts. Furthermore, First Nations peoples living on reserves in Canada are at in increased risk of having no access to running water in the home. Over 5000 families on First Nations reserves in Canada lack basic water and sewage services (7). Without fresh running water, effective hand washing may not be possible, which can increase one's risk of attaining and transmitting RSV (36). This is because the virus can be transmitted as dried respiratory secretions on surfaces such as hands which then touch the person's mouth or nose (36). Additionally, living in close quarters with numerous people can increase one's risk of obtaining any form of viral infection or disease, which can include RSV (36). Individuals who are of lower income may be forced to live in densely packed areas, which can further increase the risk of attaining RSV.

Low income may also impact breastfeeding and maternal-infant bonding. If a mother must work numerous hours in a day to maintain a living level of income, she will likely not have time to breastfeed her infant. Most newborn infants eat every 2-3 hours, 2-month-olds eat every 3-4 hours, and 6month-olds every 4-5 hours (16). It is unlikely that a mother working regular 8-hour workdays would be able to see her infant at these regular intervals for feeds. As well, approximately $16 \%$ of mothers with young children hold multiple jobs, which can further reduce the time that these mothers are able to spend with their children (9). In any circumstance, whether a child is 1 month old or 10 years old, spending less time together as a family unit can act 
as a hinderance to bonding. Low-income mothers also may not be able to afford breastfeeding assistance, such as a lactation consultant. The average hourly rate for a lactation consultant in Canada is approximately $\$ 40$ (15), which can be unaffordable for low-income families. Since lactation consultants have been defunded in numerous Canadian hospitals, low-income women may have no access to these healthcare professionals to overcome breastfeeding barriers such as RSV (3).

The Government of Canada has also recognized that being of First Nations descent is a social determinant of health (10). It has been found that First Nations and Inuit children are disproportionately affected by RSV than their Caucasian counterparts, especially on rural reserves (18). In a rural setting, the rate of RSV bronchiolitis in Inuit children living in the Baffin region of Nunavut is approximately 484 out of 1,000 infants, which is a stark difference from the rate of 27 out of 1,000 infants in urban Canada (18). Some risk factors for RSV bronchiolitis and respiratory disease in First Nations infants include exposure to second-hand tobacco smoke, overcrowding, poor indoor ventilation systems and lack of fresh running water (18). Many First Nations people, including the ones mentioned in this statistic who live on reserves are located in rural locations. Residing in a rural setting decreases access to healthcare resources, as often there are little to no healthcare professionals working in their locations. People living in rural environments may have to travel long distances to receive healthcare, which requires money and transport to do so. Many schools and institutions have attempted to increase the number of healthcare professionals working in a rural setting by running their education programs in a rural setting, but the number of healthcare professionals available in urban areas versus rural areas still remains staggering (41).

\section{Insights and Recommendations}

According to the registered Nurses Association of Ontario, if an infant is experiencing difficulty breathing as a result of respiratory illness, they should be fed small amounts of breastmilk frequently, as they tire easily (34). The solution to several of the difficulties listed above regarding breastfeeding may be simply to increase the frequency of feeds. Increased frequency of feeding in short intervals allows the infant to regain their strength in between feeds. It also ensures that while the infant is feeding, they do not become overly tired and short of breath. As well, since the child is being fed more frequently, they can still receive the same amount of nutrition as they do on a regular basis. Increased frequency should also be combined with optimal breastfeeding positioning to further enhance the feeds. The most common breastfeeding positions include the cradle, cross cradle, football carry, and side lying (31). Breastfeeding is a highly individualized experience, and while these positions are the most common, there is no specific position that will work for every mother. The main goal in breastfeeding positioning is to ensure that both the mother and the infant are in a position that reduces strain on the body and allows for a proper latch to the nipple. Mothers can breastfeed in more than one position as well. It has been found that mothers who utilize more than one position show a higher incidence of exclusive breastfeeding up to 6 months. In London, Ontario, the Middlesex London Health Unit offers free over the phone support to breastfeeding mothers, and their website pertains various videos and guidelines for breastfeeding mothers (8). This resource may be utilized by mothers experiencing difficulty in breastfeeding children with RSV. Having a trained professional available over the phone for free increases accessibility to numerous populations such as those of remote/rural areas, and those of low income. These populations may not have access to lactation consultants, especially since they are not covered by universal healthcare in Canada.

A good way to ensure that women receive some form of breastfeeding education is to provide it leading up to, and after the birth of their child in the hospital. Nurses working in labour and delivery, as well as mother-baby postpartum wards should become familiar with the baby-friendly initiative $(\mathrm{BFI})$. The $\mathrm{BFI}$ is a global program which helps mothers successfully initiate and continue to breastfeed their babies (4). Increasing women's education on how to breastfeed, and how to overcome difficulties (such as feeding an easily tired child from RSV) can decrease stress levels of the 
mother and help to facilitate long term breastfeeding. Nurses can also advocate for hospitals to adopt the BFI as a standard of practice (11). This would further regulate the education that nurses are required to receive surrounding breastfeeding and breastfeeding best practices.

There are currently no vaccinations for RSV specifically, but there are ways to prevent its occurrence. Regular hand washing, keeping hands off ones' face, avoiding close contact with sick people, covering coughs and sneezes, and cleaning and disinfecting surfaces can help protect infants from RSV (36). Additionally, palivizumab is a medication delivered through a series of shots that can help protect high risk infants from severe RSV symptoms (36). Palivizumab is a monoclonal immunoglobulin which helps to prevent exacerbations of severe symptoms from developing with RSV, thus decreasing the need for hospitalization (35). According to The American Academy of Pediatrics, palivizumab should be considered for infants who are younger than 24 months, have chronic lung disease from prematurity and have needed to receive medical treatment for this, for infants born before 32 weeks gestation, and for infants who experience risk factors such as exposure to environmental pollutants or who have a sibling who attends school (1). Breastfeeding mothers who smoke can also be encouraged to smoke after, rather than before breastfeeding their child to reduce the harmful effects of second-hand smoke as exposure to second-hand smoke is an environmental pollutant which can increase an infant's risk of respiratory illness and RSV (33). Mothers and other family members should also smoke outdoors to reduce the exposure of the child to tobacco smoke, and thus reduce the risk of developing an RSV related infection (33), (18).

Breastfeeding is listed as a recommendation to prevent RSV infection by the American Academy of Pediatrics (1). So why is it that numerous hospitals have cut funding for lactation consultants when breastfeeding can help to prevent RSV; one of the most common causes of hospitalization of infants in Canada (23)? In order for mothers to feel confident in encountering barriers in breastfeeding such as RSV, they must have resources and assistance. As stated before, lactation consultants can be expensive, and as such nurses need to advocate for their return to the hospital to increase accessibility to breastfeeding care. Nurses must voice their concerns to the government, noting that breastfeeding may help to reduce hospitalization rates of infants with lower respiratory tract infections, which can therefore save the government money.

Finally, in the case of the mother who may not be able to exclusively breastfeed her baby, there are other ways to facilitate mother-infant bonding. Many of these interventions are ones that do not require extensive intervention from healthcare professionals. For example, infant massage has been shown to help improve the maternal-infant relationship, and can be learned by watching videos of the activity on the internet (21). As well, kangaroo care is a method of holding a baby that involves skin-to-skin contact of the parents' chest to the baby's body (17). Kangaroo care has been shown to assist in maternal-infant bonding, regulate respirations in the infant, and help to increase milk supply (17), (28). As well, an increased amount of quality time spent together as early as possible in the infant's life has been shown to facilitate motherinfant bonding (2). A mother may also choose to pump milk for future use if the main issue is the infant tiring from sucking on the breast. The mother may consider pumping her breastmilk and feeding it to her infant through a bottle, which still results in the infant receiving the beneficial antibodies and nutrients from breastmilk that help to strengthen the child's immune system. Providing care to mothers in this circumstance should be done in a nonjudgemental manner. Mothers experience a great deal of societal pressure to exclusively breastfeed their child, and as described prior, they can feel guilt if they are unable to. Healthcare providers can offer assistance, but if the client does not want this, the provider must respect their decision and begin to educate these women on alternatives to breastfeeding that still facilitates proper nutrition and maternal-infant bonding.

\section{Conclusions}

RSV infection can result in numerous complications related to breastfeeding and maternalinfant bonding. The social determinants of health can contribute to higher incidence of RSV infection in infants, as well as contribute to difficulty in 
breastfeeding, bonding, and accessing resources for breastfeeding. Connecting mothers with existing educational resources for breastfeeding, advocating for more breastfeeding resources, and supporting struggling mothers with alternative ways to feed and bond with her infant with RSV can help to encourage exclusive breastfeeding and support women who require alternative means of feeding. As well, using proactive approaches such as regular hand washing and smoking outdoors help to prevent the occurrence of RSV in an infant by removing environmental risks of RSV. RSV and RSV related illnesses can be a potentially lifethreatening sickness that affects millions of infants and their breastfeeding mothers nation-wide, but with proper support mothers can become aware of their own strength and learn to overcome barriers to breastfeeding and bonding with their infants. 


\section{References}

1. American Academy of Pediatrics. Diagnosis and Management of Bronchiolitis.

PEDIATRICS. 2006; 118(4):1774-93. DOI: 10.1542/peds.2006-2223

2. Angel M. CONFERENCE ABSTRACTS: A Clinical Nurse Specialist Intervention to Improve Maternal-Infant Bonding. Clinical Nurse Specialist: The Journal for Advanced Nursing Practice. 2009; 23(2):96. Available from:

https://www.nursingcenter.com/journalarticle ?Article_ID $=844879 \&$ Journal_ID $=54033 \&$ Iss ue_ID $=844804$

3. 'As front-line as you get': Families protest cuts to breastfeeding help | CBC News [Internet]. CBCnews. CBC/Radio Canada; 2017 [cited 2021Mar2]. Available from: https://www.cbc.ca/news/canada/manitoba/l actation-consultants-protest-1.4229783

4. Baby-Friendly Initiative [Internet]. BabyFriendly Initiative Ontario. [cited 2021Mar2]. Available from: http://www.bfiontario.ca/thebaby-friendly-initiative/

5. Benoit B, Goldberg L, Campbell-Yeo M. Infant feeding and maternal guilt: The application of a feminist phenomenological framework to guide clinician practices in breast feeding promotion. Midwifery.

2015;34:58-65. DOI: 10.1016/j.midw.2015.10.011

6. Binns C, Lee MK, Low WY. The Long-Term Public Health Benefits of Breastfeeding. Asia Pacific Journal of Public Health.

2016;28(1):7-14. DOI: 10.1177/1010539515624964

7. Boyd DR. No Taps, No Toilets: First Nations and the Constitutional Right to Water in Canada. McGill Law Journal. 2011;57(1):81134. DOI: $10.7202 / 1006419 a r$

8. Breastfeeding [Internet]. Middlesex-London Health Unit. [cited 2021Mar2]. Available from: https://www.healthunit.com/breastfeeding

9. Bruns A, Pilkauskas N. Multiple Job Holding and Mental Health among Low-Income Mothers. Women's Health Issues. 2019;29(3):205-12. DOI: 10.1016/j.whi.2019.01.006
10. Canada PHAof. The Social Determinants of Health and Health Inequities [Internet]. Social determinants of health and health inequalities - Canada.ca. / Gouvernement du Canada; 2020 [cited 2021 Mar2]. Available from: https://www.canada.ca/en/publichealth/services/health-promotion/populationhealth/what-determines-health.html

11. Cardaci R. CE: Beyond Maternity Nursing: The Baby-Friendly Hospital Initiative. AJN, American Journal of Nursing. 2017;117(8):36-43. DOI: 10.1097/01.naj.0000521947.45448.d9

12. Coffman L. The NP's role in promoting and supporting breastfeeding. The Nurse Practitioner. 2019;44(3):38-42. DOI: 10.1097/01.npr.0000553401.96471.77

13. Cross J, Broad MA, Quint M, Ritson P, Thomas S. Respiratory Physiotherapy Pocketbook 3rd Edition [Internet]. 3rd ed. Amsterdam : Elsevier; 2020. Available from: https://www.elsevier.com/books/respiratoryphysiotherapy-pocketbook/cross/978-0-70205507-2

14. Geoghegan S, Erviti A, Caballero MT, Vallone F, Zanone SM, Losada JV, et al. Mortality due to Respiratory Syncytial Virus. Burden and Risk Factors. American Journal of Respiratory and Critical Care Medicine. 2017;195(1):96-103. DOI:

10.1164/rccm.201603-0658oc

15. Hourly Rate for Certification in Canada: International Board Certified Lactation Consultant (IBCLC) [Internet]. PayScale. 2021 [cited 2021Mar2]. Available from: https://www.payscale.com/research/CA/Certif ication=International_Board_Certified_Lactati on_Consultant_(IBCLC)/Hourly_Rate

16. Jain S. How Often and How Much Should Your Baby Eat? [Internet].

HealthyChildren.org. [cited 2021Mar2]. Available from: https://www.healthychildren.org/English/agesstages/baby/feeding-nutrition/Pages/HowOften-and-How-Much-Should-Your-BabyEat.aspx\#: :text=General Guidelines for Baby Feeding:\&text=Most newborns eat every 2 ,by 2 weeks of age. 
17. Kangaroo Care: What it is, Benefits \& How To Do It [Internet]. Cleveland Clinic. [cited 2021Mar2]. Available from:

https://my.clevelandclinic.org/health/treatme nts/12578-kangaroo-care

18. Kovesi T. Respiratory disease in Canadian First Nations and Inuit children. Paediatrics and Child Health. 2012; DOI:

10.1093/pch/17.7.376

19. Krause Cl. The ABCs of RSV. The Nurse Practitioner. 2018;43(9):20-6. DOI: 10.1097/01.npr.0000544277.74514.55

20. Lee W-J, Kim Y-j., Kim D-W, Lee HS, Lee HY, Kim K. Complete Genome Sequence of Human Respiratory Syncytial Virus Genotype A with a 72-Nucleotide Duplication in the Attachment Protein G Gene. Journal of Virology. 2012;86(24):13810-1. DOI: 10.1128/jvi.02571-12

21. Lindensmith R. Interventions to Improve Maternal-Infant Relationships in Mothers With Postpartum Mood Disorders. MCN: The American Journal of Maternal/Child Nursing. 2018;43(6):334-40. DOI: 10.1097/nmc.0000000000000471

22. Liu J, Leung $P$, Yang A. Breastfeeding and Active Bonding Protects against Children's Internalizing Behavior Problems. Nutrients. 2013;6(1):76-89. DOI: 10.3390/nu6010076

23. Mitchell I, Defoy I, Grubb EB. Burden of Respiratory Syncytial Virus Hospitalizations in Canada. Canadian Respiratory Journal. 2017;2017:1-9. DOI: 10.1155/2017/4521302

24. Napierkowski DB. Diagnosing and treating respiratory syncytial virus bronchiolitis. The Nurse Practitioner. 2016;41(9):1-4. DOI: 10.1097/01.npr.0000490395.74098.08

25. New York State Department of Health. Respiratory Syncytial Virus (RSV) [Internet]. Respiratory Syncytial Virus Infection (RSV). [cited 2021Mar2]. Available from: https://health.ny.gov/diseases/communicable /respiratory_syncytial_virus/fact_sheet.htm\#: :text=RSV\%20is\%20spread\%20through\%2 Ocontact,when\%20they\%20cough\%20and\% 20sneeze.
26. Pados BF, Park J, Thoyre SM, Estrem H, Nix WB. Milk Flow Rates From Bottle Nipples Used for Feeding Infants Who Are Hospitalized. American Journal of SpeechLanguage Pathology. 2015;24(4):671-9. DOI: 10.1044/2015_ajslp-15-0011

27. Paris R, Ross $O$, Molyneux L. Acute lower respiratory disease in children. Update in Anaesthesia, 251-263. Available from https://www.wfsahq.org/components/com_virt ual_library/media/132c7b556c2aef5c04363e7 69d4df39a-Acute-lower-respiratory-diseasein-children.pdf

28. Parker LA, Sullivan S, Krueger C, Kelechi T, Mueller M. Strategies to Increase Milk Volume in Mothers of VLBW Infants. MCN: The American Journal of Maternal/Child Nursing. 2013;38(6):385-90. DOI: 10.1097/nmc.0b013e3182a1fc2f

29. Piedimonte G, Perez MK. Respiratory Syncytial Virus Infection and Bronchiolitis. Pediatrics in Review. 2014;35(12):519-30. DOI: 10.1542/pir.35-12-519

30. Pisesky A, Benchimol El, Wong CA, Hui C, Crowe M, Belair M-A, et al. Incidence of Hospitalization for Respiratory Syncytial Virus Infection amongst Children in Ontario, Canada: A Population-Based Study Using Validated Health Administrative Data. PLOS ONE. 2016;11(3). DOI:

10.1371/journal.pone.0150416

31. Puapornpong $P$, Raungrongmorakot K, Manolerdtewan W, Ketsuwan S, Sinutchanan W. The Number of Infant Feeding Positions and the 6-Month Exclusive Breastfeeding Rates. J Med Assoc Thai. 2015;98(11):107581. Available from:

https://pubmed.ncbi.nlm.nih.gov/26817177/\#: : :text=The\%20relative\%20risks\%20for\%20e xclusive,\%20the\%206month\%20postpartum\%20periods.

32. Radzyminski S, Callister LC. Mother's Beliefs, Attitudes, and Decision Making Related to Infant Feeding Choices. The Journal of Perinatal Education. 2016;25(1):18-28. DOI: 10.1891/10581243.25.1.18 
33. Registered Nurse's Association of Ontario [Internet]. Module 6 Prevention and Management of Breastfeeding Problems. Breastfeeding e-learning [cited 2021Mar2]. Archived at https://elearning.rnao.ca/mod/book/view.php ?id $=790 \&$ chapterid $=59$

34. Registered Nurse's Association of Ontario [Internet]. Module 8: Infants with Special Needs. Breastfeeding e-learning [cited 2021Mar2]. Archived at https://elearning.rnao.ca/mod/book/view.php ?id $=810 \&$ chapterid $=77$

35. Robinson J, Le Saux N, Canadian Paediatric Society. Preventing hospitalizations for respiratory syncytial virus infection: Canadian Paediatric Society [Internet]. Preventing hospitalizations for respiratory syncytial virus infection | Canadian Paediatric Society. 2015 [cited 2021Mar7]. Available from: https://www.cps.ca/en/documents/position/pr eventing-hospitalizations-for-rsv-infections

36. RSV [Internet]. Centers for Disease Control and Prevention. Centers for Disease Control and Prevention; 2020 [cited 2021Mar2].

Available from:

https://www.cdc.gov/rsv/about/symptoms.ht $\mathrm{ml}$

37. Shibley-Hyde J, Else-Quest NM, Clark R. Breastfeeding, Bonding, and the MotherInfant Relationship. Merrill-Palmer Quarterly [Internet]. 20030ct [cited 2021Mar7];49(4):495-517. Available from: https://www.jstor.org/stable/23096091

38. Schweitzer JW, Justice NA. Respiratory Syncytial Virus Infection [Internet]. In: StatPearls. StatPearls Publishing, Treasure Island (FL); 2020. Available from: https://europepmc.org/article/nbk/nbk459215

39. Sriraman NK. The Nuts and Bolts of Breastfeeding: Anatomy and Physiology of Lactation. Current Problems in Pediatric and Adolescent Health Care. 2017;47(12):30510. DOI: 10.1016/j.cppeds.2017.10.001

40. Symptoms of Bronchiolitis [Internet]. NHS Choices. NHS; [cited 2021Mar2]. Available from: https://www.nhs.uk/conditions/bronchiolitis/s ymptoms/
41. Wilson R, Rourke J, Oandasan I, Bosco C. Progress made on access to rural health care in Canada. Can Fam Physician. 2020;66(1):31-6. DOI: https://www.ncbi.nlm.nih.gov/pmc/articles/PM C7012120/\#

42. Winston $R$, Chicot $R$. The importance of early bonding on the long-term mental health and resilience of children. London Journal of Primary Care. 2016;8(1):12-4. DOI: 10.1080/17571472.2015.1133012

43. Øymar K, Skjerven HO, Mikalsen IB. Acute bronchiolitis in infants, a review. Scandinavian Journal of Trauma, Resuscitation and Emergency Medicine. 2014;22(1). DOI: 10.1186/1757-7241-22-23 